\title{
Construction of a Three-Layer Directed Network Model with Multimodal Characteristics
}

\author{
Na ZHANG ${ }^{\mathrm{a}, \mathrm{b}}$, Yuzhi XIAO ${ }^{\mathrm{a}, \mathrm{b}}$, Haixing ZHAO ${ }^{\mathrm{a}, \mathrm{b}, 1}$, Cunyang TANG $^{\mathrm{a}, \mathrm{b}}$ and \\ Baoyang CUI ${ }^{\mathrm{a}, \mathrm{b}}$ \\ ${ }^{a}$ College of Computer, Qinghai Normal University, Xining, China \\ b The State Key Laboratory of Tibetan Intelligent Information Processing and \\ Application, Xining, Qinghai 810008, China
}

\begin{abstract}
The multi-layer directed network model focuses on portraying the directionality, diversity and difference of the edges in the network. It is also one of the powerful tools for analyzing the complexity of the network system and the heterogeneous interaction characteristics between network layers. This paper constructs three three-layer directed network models combined with traditional complex network theory and analyzes their degree distribution characteristics. By controlling the out-degree and in-degree of the middle-level nodes, a network structure with multi-peak characteristics is configured. Combined with numerical simulation, it is concluded that the number of links between the layers of the directed network makes the network characterize the characteristics of unimodal, bimodal, and trimodal; the combination of the optimal mechanism and the changes in the number of inter-layer links, the network characteristics show the coexistence of power law and unimodal. The research results of this paper have practical value for the analysis of network generation mechanism and correlation law by using multilayer directed network theory in the era of big data.
\end{abstract}

Keywords. Complex network, multi-layer directed network, degree distribution, multi-peak characteristics, hierarchical connection.

\section{Introduction}

The development of network science has become more mature, and the research on complex networks has become more in-depth. With the expansion of network scale and the complexity of application scenarios in real society, network models have gradually shifted to interactive coupling networks, multi-layer networks or super networks. Among them, the multi-layer network is gradually applied to multiple real system structure model references with its high flexibility, multi-attribute hierarchical relationship and the diversity of connection types [1-6].

The structural characteristics of multi-layer networks are very important for the study of complex system dynamics. The construction of multi-layer network models is the prerequisite for exploring the characteristics of network structures. There are two

\footnotetext{
${ }^{1}$ Corresponding Author, Haixing ZHAO, College of Computer, Qinghai Normal University, Xining, China; E-mail: h.x.zhao@163.com.
} 
general methods for building multi-layer network models: one is increasing multi-layers network model, the network scale continues to expand, and there is a generalized priority connection principle; The other is a multi-layer network set with structural constraints, which has a fully controlled correlation and overlap set [7-11]. Chen [12] et al. constructed a subway-bus two-layer network model, combined with the identification method of important nodes in the complex network theory, studied the best optimized node location and optimized number of the existing network, and proved the effectiveness of the method sex. Shen [13] et al. built a multi-layer supply chain financial network, and explored the structure of the supply chain financial network, cumulative distribution and other issues. The study obtained the degree of dependence between the levels of the supply chain financial network and the number of connections of new nodes, changing the size of the network's scaling law affects the number of hub nodes. In [14] constructed a two-layer ER network and a two-layer BA network, and studied the influence of the correlation between nodes on the robustness of the network. It is concluded that the mutual coupling characteristics of the multilayer network will affect the robust performance of the network. In [15] proposed and constructed three threelayer complex network models to quantitatively describe the inter-layer dependencies of the multi-layer network with random probabilities. The experimental results show that different random probabilities characterize the three-layer network with characteristics such as single peak, double peak and triple peak.

The directionality of connecting edges in the real network cannot be ignored. The out-degree and in-degree of the directed network reflect the influence and adaptability of the network [16]. Liu Dawei [17] et al. proposed a method for generating a directed complex network model by analyzing the local structure of a directed network, and used the corresponding link prediction algorithm to test the generated model, and verified the effectiveness of the network model with real data sets. Chen Xiao [18] et al. explored the construction mechanism of Weibo forwarding network and proposed a directed weighted network model. Theoretical analysis and simulation experiments show that the intensity distribution, degree distribution, and intensity-degree correlation of the model obey the power law. Distribution, with the characteristics of high clustering and short path. At the same time, the actual data of Weibo forwarding is collected to verify the correctness of the model. Liu Dahai [19] et al. proposed a directed community discovery algorithm for multi-dimensional complex networks based on directed community discovery and fusion of information from multiple dimensions. Combining real data sets to verify the effectiveness of the algorithm, it is consistent with existing algorithms. In comparison, the accuracy rate, recall rate and F-measure value have been improved to a certain extent.

In a multi-layer directed network, different connection directions between nodes have different effects on the characteristics of the network structure. Based on the common sense that most human infectious diseases originate from animals, and that zoonotic diseases are always spread through directional interconnected networks, $\mathrm{Xu}$ Zhongpu [20] et al. considered the epidemic dynamics of zoonotic diseases on a oneway cyclic coupling network. Combined with the heterogeneous mean field method, two unidirectional three-layer circular interactive networks are constructed. One model describes the direct connection between the interactive networks, and the other model describes the diseases that are transmitted through the vectors between the interactive networks. Combined with theoretical analysis, the basic reproduction number of the model and the stability of the equilibrium point are obtained. There are few existing research results on multi-layer directed networks, and most of the network models are 
constructed based on real data sets, which lack universality. Therefore, this paper constructs three three-layer directed networks. The number of directed links constructs a network with multi-peak characteristics. The influence of the number of directed links between layers on the network degree distribution is discussed.

\section{Introduction To Basic Concepts}

A multi-layer directed network is a complex network structure composed of $\mathrm{M}$ directed network layers through inter-layer directed connections. A multi-layer directed networks a pair $\overrightarrow{\mathcal{M}}=\{\vec{G}, \vec{C}\}$ where $\vec{G}=\left\{\overrightarrow{G_{\alpha}} ; \alpha \in\{1, \ldots, M\}\right\}, \overrightarrow{G_{\alpha}}=\left(V_{\alpha}, \overrightarrow{E_{\alpha}}\right)$ is directed network layer. $\vec{C}=\left\{\overrightarrow{E_{\alpha \beta}} \in V_{\alpha} \times V_{\beta} ; \alpha, \beta \in\{1, \ldots, M\}, \alpha \neq \beta\right\}$ represents the set of directed edges connected to the directed network layer. $\overrightarrow{E_{\alpha}}$ represents the interconnection inside the directed network layer, $\overrightarrow{E_{\alpha \beta}}$ represents the connection between multiple layers of directed network layers. Use $V_{\alpha}=\left\{v_{1}^{\alpha}, v_{2}^{\alpha}, \ldots, v_{N \alpha}^{\alpha}\right\}$ represents a collection of nodes in the directed network layer $\overrightarrow{G_{\alpha}} \cdot A^{[\alpha]}=\left(a_{i j}^{\alpha} \in R^{N_{\alpha} \times N_{\alpha}}\right)$ represents the adjacency matrix of each individual directed network layer, and

$$
a_{i j}^{\alpha}=\left\{\begin{array}{cc}
1, & i f\left(v_{i}^{\alpha}, v_{j}^{\alpha}\right) \in \overrightarrow{E_{\alpha}}, \\
0, & \text { otherwise, }
\end{array}\right.
$$

for $1 \leq i, j \leq N_{\alpha}$ and $1 \leq \alpha \leq M$. The interlayer adjacency matrix corresponding to $\overrightarrow{E_{\alpha \beta}}$ is the matrix $A^{[\alpha, \beta]}=\left(a_{i j}^{\alpha \beta}\right) \in R^{N_{\alpha} \times N_{\beta}}$ given by:

$$
a_{i j}^{\alpha \beta}=\left\{\begin{array}{cc}
1, & i f\left(v_{i}^{\alpha}, v_{j}^{\beta}\right) \in \overrightarrow{E_{\alpha \beta}}, \\
0 & \text { otherwish, }
\end{array}\right.
$$

The projection network of $\overrightarrow{\mathcal{M}}$ is the graph $\operatorname{proj}(\overrightarrow{\mathcal{M}})=\left(\mathrm{V}_{\overline{\mathcal{M}}}, \mathrm{E}_{\overrightarrow{\mathcal{M}}}\right)$ where

$$
\begin{gathered}
V_{\overline{\mathcal{M}}}=\bigcup_{\alpha=1}^{M} V_{\alpha} \\
E_{\overline{\mathcal{M}}}=\left(\bigcup_{\alpha=1}^{M} \overrightarrow{E_{\alpha}}\right) \cup\left(\bigcup_{\alpha, \beta=1, \alpha \neq \beta}^{M} \overrightarrow{E_{\alpha \beta}}\right) .
\end{gathered}
$$

\section{Construction Of Multi-Layer Directed Network Model}

The diversity and selectivity of the multi-layer directed network structure makes the complex network system more detailed and universal. It can vividly describe the interdependent connection between different groups with selectivity, and there will be a multi-layer complex network. The model is more specific and detailed. The multi-layer directed network model construction algorithm is based on the directed random connection algorithm, the directed scale-free connection algorithm, and the directed 
small world connection algorithm. The number of links between layers is controlled by the connection probability. The specific construction process is as follows:

\subsection{Three-Layer Directed Random Network (Tde) Construction Process}

(1) Initialization: Define a single-layer directed network as $\overrightarrow{G_{\alpha}}, \overrightarrow{G_{\beta}}, \overrightarrow{G_{\gamma}}$. The probability of intra-layer connection is $p_{1} \in(0,1)$, and the probability of inter-layer connection is $p_{\alpha \beta} \in(0,1)$ and $p_{\beta \gamma} \in(0,1)$, the number of nodes is $\left|V_{\alpha}\right|=n$, the number of connections within the layer is $\left|\overrightarrow{E_{\alpha}}\right|=p_{1} \times n \times(n-1)$, the same goes for the other individual layers. The number of connected edges between layers is $\left|\overrightarrow{E_{\alpha \beta}}\right|=2 n^{2} p_{\alpha \beta}$ or $\left|\overrightarrow{E_{\beta \gamma}}\right|=2 n^{2} p_{\beta \gamma}$.

(2) Directed edges within the layer: Randomly select $\mathrm{S}$ pairs of different vertex sets $\left\{v_{x}^{\alpha}, v_{y}^{\alpha}\right\}$ and $\left\{v_{y}^{\alpha}, v_{x}^{\alpha}\right\}$ as different vertex pairs, take a pair of vertices $\left\{v_{x}^{\alpha}, v_{y}^{\alpha}\right\}, v_{x}^{\alpha}$ as the arc tail and $v_{y}^{\alpha}$ as the arc head to form a directed edge. Repeat the above operation for the randomly selected $S$ pairs of different vertex sets to generate $\left|\overrightarrow{E_{\alpha}}\right|$ directed edges, thus generating directed network $\overrightarrow{G_{\alpha}}$, the construction algorithm of directed networks $\overrightarrow{G_{\beta}}$ and $\overrightarrow{G_{\gamma}}$ is also shown above.

(3) Directed edges between layers: Given the probability $p_{\alpha \beta} \in(0,1)$ and $p_{\beta \gamma} \in(0,1)$ of connection edges between layers, limit the number of directed connections between layers to $\left|\overrightarrow{E_{\alpha \beta}}\right|=2 n^{2} p_{\alpha \beta}$ or $\left|\overrightarrow{E_{\beta \gamma}}\right|=2 n^{2} p_{\beta \gamma}$, and then select one node in each of the directed networks $\overrightarrow{G_{\alpha}}$ and $\overrightarrow{G_{\beta}}$ to form a node pair, and specify the pair of nodes are connected in a direction until the number of connected edges reaches the limit, and the algorithm ends. In the same way, the connecting edges between directed networks $\overrightarrow{G_{\beta}}$ and $\overrightarrow{G_{\gamma}}$ are also operated as above.

(4) Marked network: The three-layer directed random network obtained by the above algorithm is marked as TDE, which is convenient to distinguish other types of threelayer directed networks.

Through the above construction process, the time complexity of constructing the directed network within the layer is $O\left(n^{2}\right)$, and the time complexity of the interlayer is $O\left(n^{2}\right)$, so the time complexity of the three-layer directed network is $O\left(n^{2}\right)$. 


\subsection{Three-Layer Directed Scale-Free Network (Tdb) Construction Process}

(1) Initialization: Define a single-layer directed network as $\overrightarrow{G_{\alpha}}, \overrightarrow{G_{\beta}}, \overrightarrow{G_{\gamma}}$. Each time a new node is added, $\mathrm{m}$ directed connections are added. The probability of inter-layer connections and the number of inter-layer directed connections are the same as the threelayer directed random network.

(2) Directed edges within the layer: Given the number $n$ of vertices and the number $m$ of edges added each time a point is added, a one-way ring with $2 \times m-1$ vertex is first constructed, add $\mathrm{m}$ edges at a time, randomly select $\mathrm{m}$ vertices in the original network to connect edges by the roulette wheel selection, among them, $m / 2$ directed edges take $v_{x}^{\alpha}$ as the arc tail, and $m / 2$ directed edges take $v_{x}^{\alpha}$ as the arc head; If $\mathrm{m}$ is an odd number, $(m-1) / 2$ directed edges take $v_{x}^{\alpha}$ as the arc end, $(m-1) / 2$ directed edges take $v_{x}^{\alpha}$ as the arc head, and the remaining edges that need to be added are randomly selected to be $v_{x}^{\alpha}$ as the arc head or arc end, until the network vertex scale reaches $\mathrm{n}$. Thus generating directed network $\overrightarrow{G_{\alpha}}$, the construction algorithm of directed networks $\overrightarrow{G_{\beta}}$ and $\overrightarrow{G_{\gamma}}$ is also shown above.

(3) The connecting edges between layers are the same as shown in the TDE (3) algorithm. (4) Marked network: The three-layer directed scale-free network obtained by the above algorithm is marked as TDB, which is convenint to distinguish other types of three-layer directed networks.

Through the above construction process, the time complexity of constructing the directed network within the layer is $O\left(n^{2}\right)$, and the time complexity of the interlayer is $O\left(n^{2}\right)$, so the time complexity of the three-layer directed network is $O\left(n^{2}\right)$.

\subsection{Three-Layer Directed Small World Network(Tdw) Construction Process}

(1)Initialization: Define a single-layer directed network as $\overrightarrow{G_{\alpha}}, \overrightarrow{G_{\beta}}, \overrightarrow{G_{\gamma}}$, probability of reconnection is $p_{1} \in(0,1)$, the probability of inter-layer connection and the number of inter-layer directed connections are the same as the three-layer directed random network. (2) Directed edges within the layer: Given the number of vertices $n$ and the value $k$, first construct a ring-shaped k-rule directed network with $\mathrm{n}$ vertices. Each vertex in the network is connected to the $\mathrm{k}$ vertices on the right end, form a directed edge with this point as the end of the arc. At the same time, according to the given reconnection probability, the number of reconnections becomes $p_{1} \times k \times n$. Traverse all the edges in the network, randomly select $p_{1} \times k \times n$ directed edges, then randomly select the arc heads or arc tails of these directed edges, and randomly select the remaining vertices in the network to form new directed edges (the directed edges after reconnection cannot be 
double edges or self-loops). Thus generating directed network $\overrightarrow{G_{\alpha}}$, the construction algorithm of directed networks $\overrightarrow{G_{\beta}}$ and $\overrightarrow{G_{\gamma}}$ is also shown above.

(3) The connecting edges between layers are the same as shown in the TDE (3) algorithm. (4) Marked network: The three-layer directed small world network obtained by the above algorithm is marked as TDW, which is convenient to distinguish other types of threelayer directed networks.

Through the above construction process, the time complexity of constructing the directed network within the layer is $O(n)$, and the time complexity of the interlayer is $O\left(n^{2}\right)$, so the time complexity of the three-layer directed network is $O\left(n^{2}\right)$.

\section{Three-Layer Directed Network Characteristics Analysis}

The number of nodes in each layer of the three-layer directed network is $n=500$, the total number of nodes is $N=1500$. Table 1 introduces the definition of relevant parameters for the construction of the three-layer directed network model.

Table 1. Three-layer directed network construction parameter definition.

\begin{tabular}{cc}
\hline Symbol & Meaning \\
\hline$p_{1} / m$ & In-layer connection probability or increase the number of edges \\
$p_{\alpha \beta}\left(p_{\beta \gamma}\right)$ & Interlayer connection probability \\
$\left\langle k_{\alpha}^{\text {in }}\right\rangle$ & $\alpha$ layer average in-degree \\
$\left\langle k_{\alpha}^{\text {out }}\right\rangle$ & $\alpha$ layer average out-degree \\
$\left\langle k_{\beta}^{\text {in }}\right\rangle$ & $\beta$ layer average in-degree \\
$\left\langle k_{\beta}^{\text {out }}\right\rangle$ & $\beta$ layer average out-degree \\
$\left\langle k_{\gamma}^{\text {in }}\right\rangle$ & $\gamma$ layer average in-degree \\
$\left\langle k_{\gamma}^{\text {out }}\right\rangle$ & $\gamma$ layer average out-degree \\
$\left\langle k^{\text {in }}\right\rangle$ & Average in-degree of three-layer directed network \\
$\left\langle k^{\text {out }}\right\rangle$ & Average out-degree of three-layer directed network \\
\hline
\end{tabular}

\subsection{Three-Layer Directed Network Degree Distribution}

Degree distribution is one of the indicators to describe the network structure. Use $P(k)$ to describe the degree distribution of nodes in the network, and $P(k)$ is defined as the probability of a randomly selected node in the network with degree $\mathrm{k}$, where $P(k)=N_{k} / N \cdot N$ : total number of network nodes. $N_{k}$ : number of nodes of degree 
k. This paper analyzes the network topology characteristics based on the directed network, so the global network degree distribution is divided into in-degree distribution and out-degree distribution. The in-degree distribution $P\left(k^{i n}\right)$ of a directed network is defined as the probability of a randomly selected node in the network with an in-degree of $k^{i n}$, and the out-degree distribution $P\left(k^{\text {out }}\right)$ of a directed network is defined as the probability of a randomly selected node in the network with an in-degree of $k^{\text {out }}$.

Due to the complex inter-layer connection mechanism of the multi-layer directed network, different inter-layer connection probabilities will cause the multi-layer directed network to show different degree distribution types. By controlling the hierarchical connection probability, the degree distribution characteristics of the network are explored, and the experimental design Determine two sets of different inter-layer connection probabilities to explore the degree distribution behavior, which are the same probability of connecting edges between control layers and different probability of connecting edges between control layers.

\subsubsection{The Probability of Directed Edges Between Layers is the Same.}

The inter-layer connection probability is the same. Set two sets of experiments. One is the parameter control whose sum of the intra-layer connection probability and the interlayer connection probability is 1 , and the other is the degree distribution when the intralayer connection probability and the inter-layer connection probability are the same.

(1)When $p_{1}+p_{\alpha \beta}\left(p_{\beta \gamma}\right)=1$ and $p_{\alpha \beta}=p_{\beta \gamma}, p_{1} \in(0,1)$ and $p_{\alpha \beta}\left(p_{\beta \gamma}\right) \in(0,1)$, the number of nodes in each layer of the three-layer directed network is $n=500$, the total number of nodes is $N=1500$. The out-degree distribution and in-degree distribution of three three-layer networks are analyzed, and the experimental results are shown in table 2 and figure 1.

Table 2. Degree distribution of three kinds of three-layer directed networks.

\begin{tabular}{|c|c|c|c|c|c|c|}
\hline Pyne $\quad$ Parameter & \multicolumn{2}{|c|}{ Network TDE } & \multicolumn{2}{|c|}{ Network TDB } & \multicolumn{2}{|c|}{ Network TDW } \\
\hline$p_{1} / m$ & 0.1 & 0.99 & 2 & 2 & 0.1 & 0.99 \\
\hline$p_{\alpha \beta}\left(p_{\beta \gamma}\right)$ & 0.9 & 0.01 & 0.001 & 0.9 & 0.9 & 0.01 \\
\hline$\left\langle k_{\alpha}^{\text {in }}\right\rangle$ & 499.9 & 499.1 & 2.5 & 452.4 & 452.1 & 7 \\
\hline$\left\langle k_{\alpha}^{\text {out }}\right\rangle$ & 499.86 & 498.9 & 2.5 & 451.5 & 451.9 & 7 \\
\hline$\left\langle k_{\beta}^{i n}\right\rangle$ & 949.8 & 503.9 & 2.9 & 901.4 & 902 & 12 \\
\hline$\left\langle k_{\beta}^{\text {out }}\right\rangle$ & 949.99 & 504.1 & 3.0 & 902.5 & 902 & 12 \\
\hline$\left\langle k_{\gamma}^{i n}\right\rangle$ & 499.95 & 499 & 2.5 & 452.1 & 451.9 & 7 \\
\hline$\left\langle k_{\gamma}^{\text {out }}\right\rangle$ & 499.85 & 499 & 2.5 & 451.9 & 452.1 & 7 \\
\hline$\left\langle k^{i n}\right\rangle$ & 649.9 & 500.7 & 2.7 & 602 & 602 & 8.7 \\
\hline$\left\langle k^{\text {out }}\right\rangle$ & 649.9 & 500.7 & 2.7 & 602 & 602 & 8.7 \\
\hline Degree distribution & bimodal & unimodal & power law & bimodal & bimodal & unimodal \\
\hline
\end{tabular}


Analyzing table 2 and figure 1, we can get that when $p_{1}=0.99$ and $p_{\alpha \beta}\left(p_{\beta \gamma}\right)=0.01$, for single-layer directed networks $\overrightarrow{G_{\alpha}}, \overrightarrow{G_{\beta}}, \overrightarrow{G_{\gamma}}$, the average outdegree and average in-degree values are not much different. At this time, the out-degree distribution and in-degree distribution of the three-layer directed network TDE and the three-layer directed network TDW all show unimodal characteristics, and the out-degree distribution and in-degree distribution of the three-layer directed network TDB show power-law characteristics. Because the value of the inter-layer connection probability has a greater impact on the middle layer, when the value of $p_{\alpha \beta}\left(p_{\beta \gamma}\right)$ increases and approaches 0.9 , the average out-degree and average in-degree values of the single layer $\overrightarrow{G_{\beta}}$ are larger than those of other layers There are many, so the superposition of the values of $<k_{\alpha}^{\text {in }}>$ and $<k_{\gamma}^{\text {in }}>\left(<k_{\alpha}^{\text {out }}>\right.$ and $\left.<k_{\gamma}^{\text {out }}>\right)$ forms a peak, and the value of $<k_{\beta}^{\text {in }}>\left(<k_{\beta}^{\text {out }}>\right)$ forms another peak. Therefore, the out-degree distribution and indegree distribution of the network TDE and the network TDW have changed from a unimodal characteristic to a bimodal characteristic, and the out-degree distribution and in-degree distribution of the network TDB have also changed from a power law distribution to a bimodal distribution.
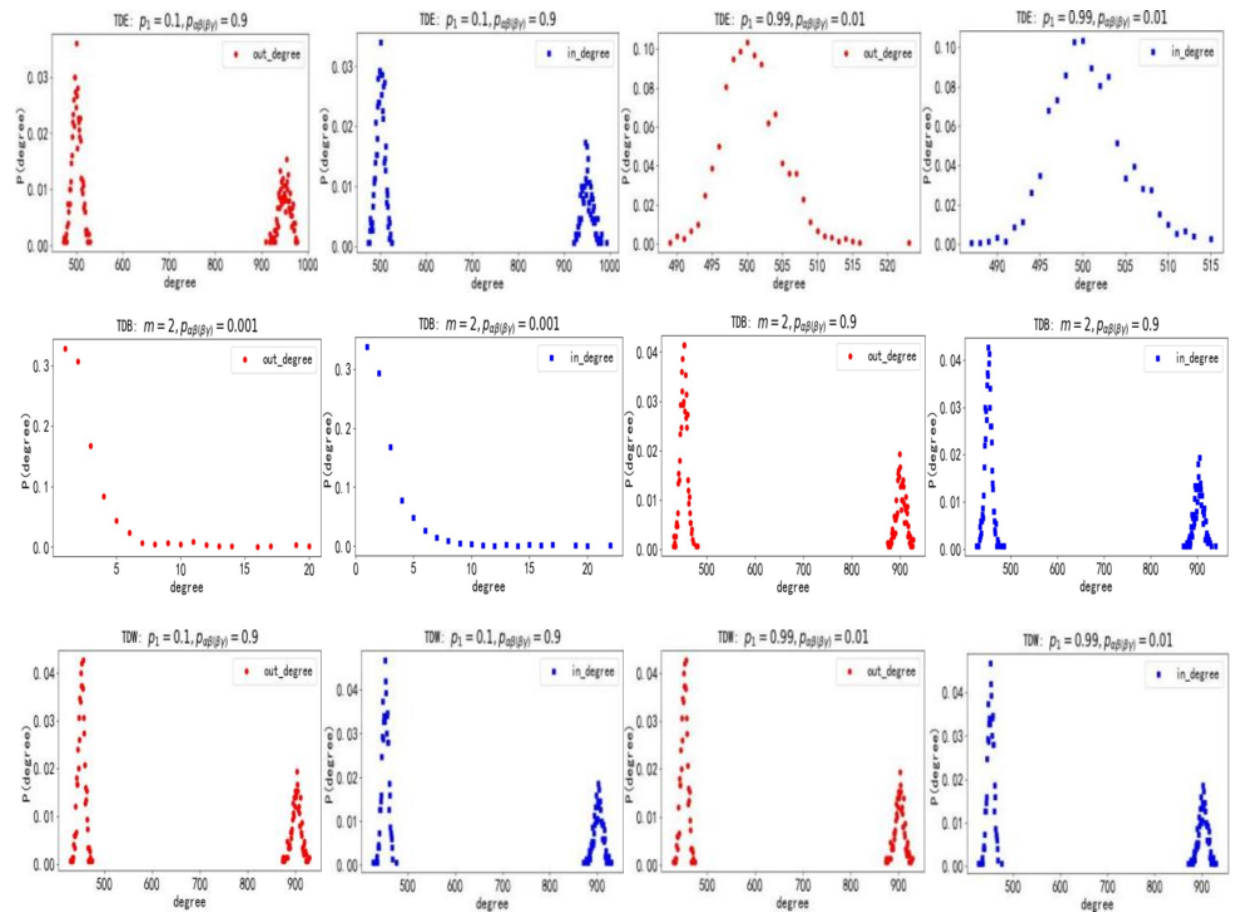

Figure 1. Degree distribution of three kinds of three-layer directed networks.

(2)When $p_{1}=p_{\alpha \beta}\left(p_{\beta \gamma}\right)$ and $p_{1} \in(0,1)$ and $p_{\alpha \beta}\left(p_{\beta \gamma}\right) \in(0,1)$. In order to analyze the network degree distribution characteristics more clearly, we will discuss the three types of networks separately. 


\section{1) Degree distribution of network TDE}

When the inter-layer connection probability and the inter-layer connection probability are the same, as the probability value increases, the degree distribution behavior of the network is explored. When the connection probability is set to $p_{1}=p_{\alpha \beta}\left(p_{\beta \gamma}\right)=0.01,0.1,0.3,0.5,0.7$, the experimental results are shown in table 3 and figure 2 .

Table 3. Degree distribution of three-layer directed network TDE.

\begin{tabular}{c|ccccccccccc}
\hline Parameter & $p_{1}$ & $\begin{array}{l}p_{\alpha \beta} \\
\left(p_{\beta \gamma}\right)\end{array}$ & $\left\langle k_{\alpha}^{\text {in }}\right\rangle$ & $\left\langle k_{\alpha}^{\text {out }}\right\rangle$ & $\left\langle k_{\beta}^{\text {in }}\right\rangle$ & $\left\langle k_{\beta}^{\text {out }}\right\rangle$ & $\left\langle k_{\gamma}^{\text {in }}\right\rangle$ & $\left\langle k_{\gamma}^{\text {out }}\right\rangle$ & $\left\langle k^{\text {in }}\right\rangle$ & $\left\langle k^{\text {out }}\right\rangle$ & $\begin{array}{c}\text { Degree } \\
\text { Distribution }\end{array}$ \\
\hline & & & & & & & & & & & \\
Nype & 0.01 & 0.01 & 9.9 & 10 & 15 & 14.97 & 10 & 9.96 & 11.66 & 11.66 & unimodal \\
TDE & 0.1 & 0.1 & 100 & 99.67 & 150 & 149.7 & 99.4 & 100.4 & 116.6 & 116.6 & bimodal \\
& 0.3 & 0.3 & 299.8 & 299.6 & 449 & 450 & 300 & 299 & 349.7 & 349.7 & bimodal \\
& 0.5 & 0.5 & 500 & 499 & 749.6 & 749.4 & 498.9 & 500 & 582.8 & 582.8 & bimodal \\
& 0.7 & 0.7 & 699 & 699.6 & 1050 & 1049 & 699 & 699.6 & 815.95 & 815.95 & bimodal \\
\hline
\end{tabular}

Observing table 3 and figure 2, we can get that when $p_{1}=p_{\alpha \beta}\left(p_{\beta \gamma}\right)=0.01$, the average out-degree and average in-degree values of a single layer are basically the same, so the out-degree distribution and in-degree distribution show unimodal characteristics. The increase of $p_{1}$ and $p_{\alpha \beta}\left(p_{\beta \gamma}\right)$ makes the average out-degree and average in-degree value of the network also increase. In this process, the average out-degree and average in-degree value of the individual layers $\overrightarrow{G_{\alpha}}$ and $\overrightarrow{G_{\gamma}}$ are basically the same, and the average out-degree and average in-degree value of the individual layer $\overrightarrow{G_{\beta}}$ the average in-degree value has increased significantly. Therefore, the out-degree distribution and in-degree distribution of the network show bimodal characteristics, and the larger the value of $p_{\alpha \beta}\left(p_{\beta \gamma}\right)$, the larger the gap between the two peaks.

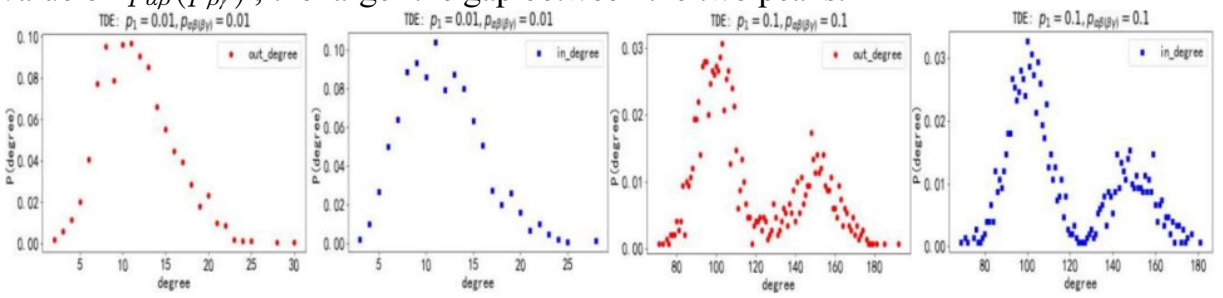




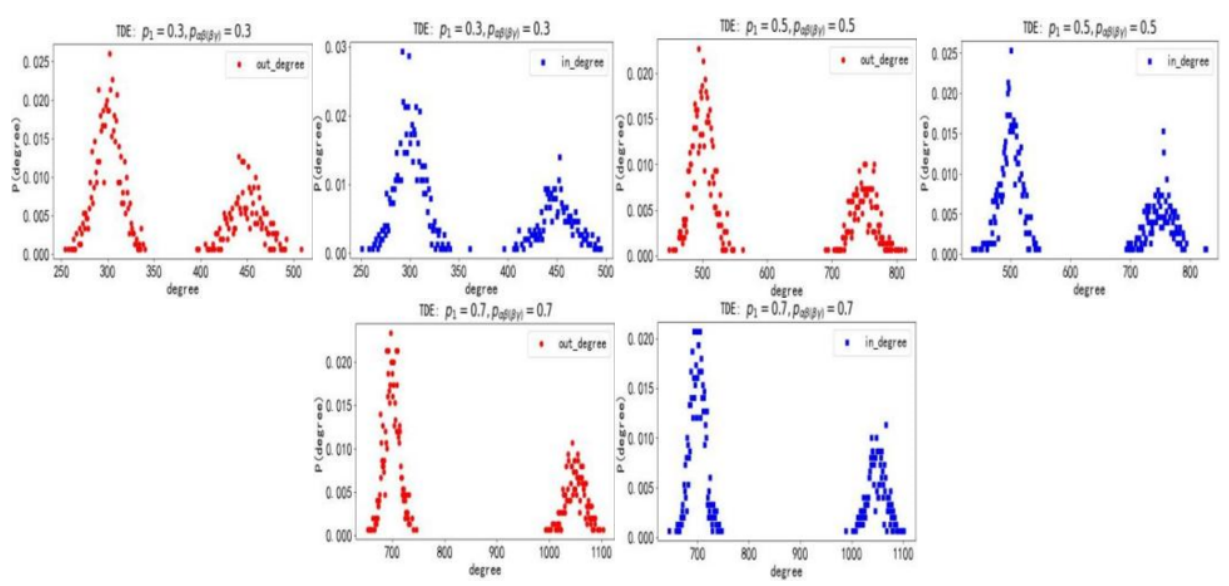

Figure 2. Degree distribution of three-layer directed network TDE.

2) Degree distribution of network TDB

The nodes of the network layer are connected by the optimal non-scale algorithm, and the nodes between the layers are connected by the random connection algorithm. The TDB network only considers the changes in the probability of the edges of the layers. The experimental results are shown in table 4 and figure 3.

It can be seen from table 4 and figure 3 that when the inter-layer connection probability approaches 0.001 , the average out-degree and average in-degree values of the directed networks $\overrightarrow{G_{\alpha}}$ and $\overrightarrow{G_{\gamma}}$ of the single layer are basically the same, and the average out-of-degree values of the directed network $\overrightarrow{G_{\beta}}$ in the single layer are basically the same. The degree and average in-degree values are slightly larger than those of the other two separate layers. At this time, the out- and in-degree distribution of the network TDB presents a power-law distribution; When the inter-layer connection probability approaches 0.01 , the out-degree distribution and in-degree distribution of the network TDB evolve from a power-law distribution to a unimodal characteristic; As the probability of inter-layer connection increases, the average out-degree and average indegree values of the directed networks $\overrightarrow{G_{\alpha}}$ and $\overrightarrow{G_{\beta}}$ and $\overrightarrow{G_{\gamma}}$ of the individual layers also increase. During the increasing period, the average of the directed networks $\overrightarrow{G_{\alpha}}$ and $\overrightarrow{G_{\gamma}}$ in the individual layers the out-degree and average in-degree values are always basically the same. The average out-degree and in-degree values of the directed network $\overrightarrow{G_{\beta}}$ of a single layer increase by a relatively large extent. Therefore, the out-degree distribution and in-degree distribution of the network have evolved from a unimodal characteristic to a bimodal characteristic, and the larger the value of $p_{\alpha \beta}\left(p_{\beta \gamma}\right)$, the larger the gap between the double peaks. 
Table 4. Degree distribution of three-layer directed network TDB.

\begin{tabular}{|c|c|c|c|c|c|c|c|c|c|c|c|}
\hline Parameter & $m$ & $\begin{array}{l}p_{\alpha \beta} \\
\left(p_{\beta \gamma}\right)\end{array}$ & $\left\langle k_{\alpha}^{i n}\right\rangle$ & $\left\langle k_{\alpha}^{\text {out }}\right\rangle$ & $\left\langle k_{\beta}^{i n}\right\rangle$ & $\left\langle k_{\beta}^{\text {out }}\right\rangle$ & $\left\langle k_{\gamma}^{i n}\right\rangle$ & $\left\langle k_{\gamma}^{\text {out }}\right\rangle$ & $\left\langle k^{i n}\right\rangle$ & $\left\langle k^{\text {out }}\right\rangle$ & $\begin{array}{c}\text { Degree } \\
\text { Distribution }\end{array}$ \\
\hline \multirow{5}{*}{$\begin{array}{l}\text { Network } \\
\text { TDB }\end{array}$} & \multirow{5}{*}{2} & 0.001 & 2.5 & 2.5 & 2.98 & 3.01 & 2.48 & 2.51 & 2.66 & 2.66 & power law \\
\hline & & 0.01 & 7.02 & 6.97 & 11.94 & 12.05 & 7.02 & 6.97 & 8.66 & 8.66 & unimodal \\
\hline & & 0.1 & 51.77 & 52.22 & 101.8 & 102 & 52.4 & 51.6 & 68.66 & 68.66 & bimodal \\
\hline & & 0.3 & 151.8 & 152.2 & 301.8 & 302.2 & 152.4 & 151.6 & 202 & 202 & bimodal \\
\hline & & 0.5 & 252.2 & 251.8 & 501.9 & 502 & 251.9 & 252.1 & 335.33 & 335.33 & bimodal \\
\hline
\end{tabular}
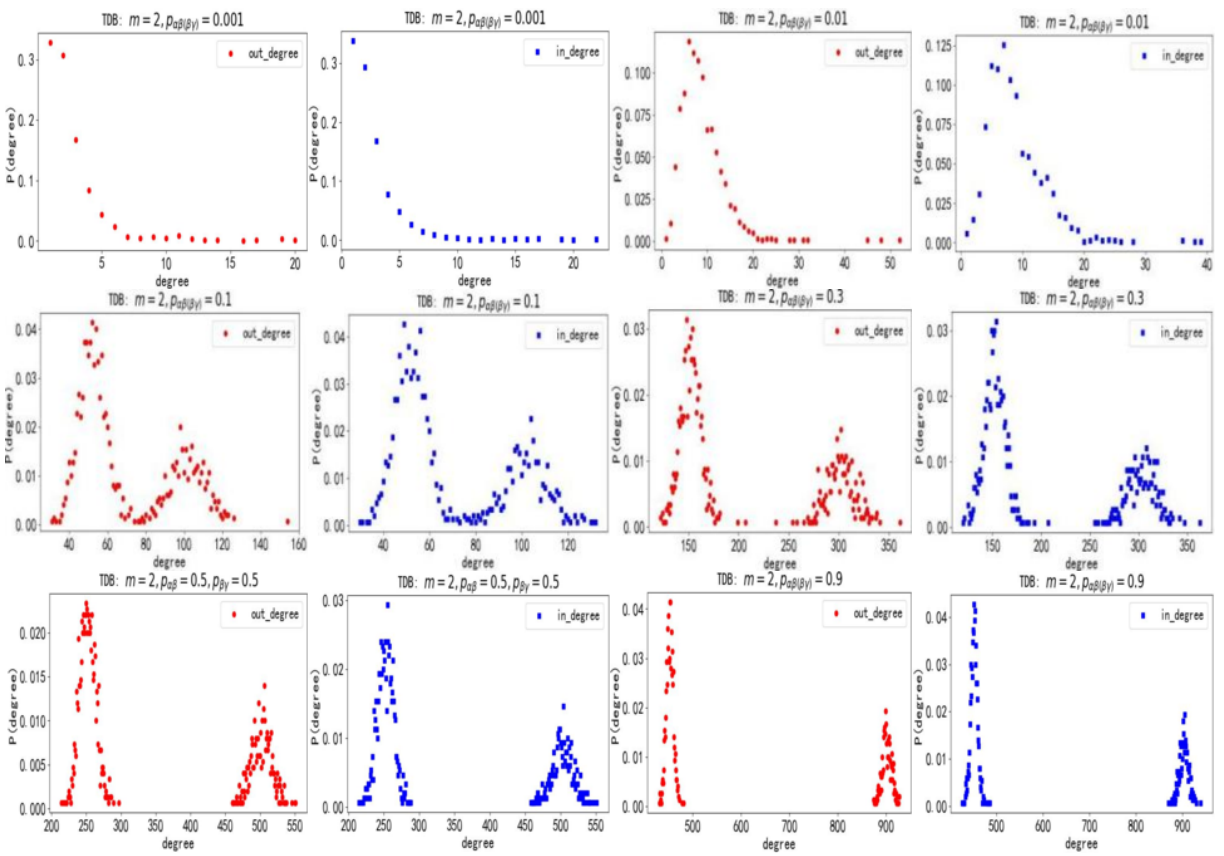

Figure 3. Degree distribution of three-layer directed network TDB.

3) Degree distribution of network TDW

Exploring the degree distribution of the three-layer directed network TDW, the value range of $p_{1}$ and $p_{\alpha \beta}\left(p_{\beta \gamma}\right)$ is $(0,1)$, and the experimental results are shown in table 5 and figure 4 . 
Table 5. Degree distribution characteristics of three-layer directed network TDW.

\begin{tabular}{c|ccccccccccc}
\hline Parameter & \multicolumn{1}{c}{$\begin{array}{c}p_{1} \\
\text { Type }\end{array}$} & $\left.\begin{array}{c}p_{\alpha \beta} \\
p_{\beta \gamma}\end{array}\right)$ & $\left\langle k_{\alpha}^{\text {in }}\right\rangle$ & $\left\langle k_{\alpha}^{\text {out }}\right\rangle$ & $\left\langle k_{\beta}^{\text {in }}\right\rangle$ & $\left\langle k_{\beta}^{\text {out }}\right\rangle$ & $\left\langle k_{\gamma}^{\text {in }}\right\rangle$ & $\left\langle k_{\gamma}^{\text {out }}\right\rangle$ & $\left\langle k^{\text {in }}\right\rangle$ & $\left\langle k^{\text {out }}\right\rangle$ & $\begin{array}{c}\text { Degree } \\
\text { Distribution }\end{array}$ \\
\hline \multirow{2}{*}{$\begin{array}{c}\text { Network } \\
\text { TDW }\end{array}$} & 0.01 & 0.01 & 6.9 & 7.1 & 12 & 11.88 & 7 & 7 & 8.65 & 8.65 & unimodal \\
& 0.1 & 0.1 & 51.8 & 52.2 & 102.1 & 101.9 & 52.09 & 52 & 68.65 & 68.65 & bimodal \\
& 0.3 & 0.3 & 151.9 & 152.1 & 301.7 & 302.3 & 152.4 & 151.6 & 202 & 202 & bimodal \\
& 0.5 & 0.5 & 251.9 & 252.1 & 502.7 & 501.2 & 251.4 & 252.6 & 335.3 & 335.3 & bimodal \\
& 0.9 & 0.9 & 451.9 & 452.1 & 902.5 & 901.5 & 451.6 & 452.4 & 602 & 602 & bimodal \\
\hline
\end{tabular}

Analyzing table 5 and figure 4 , it can be seen that when the inter-layer connection probability within a layer tends to 0.01 , the network out-degree distribution and in-degree distribution show a unimodal characteristic. The average out-degree and average indegree value increases with the inter-layer connection probability in the layer Increase and increase. At this time, the network out-degree distribution and in-degree distribution are bimodal, and because the difference between the average out-degree and the average in-degree value is large, the distance between the two peaks is getting farther and farther.
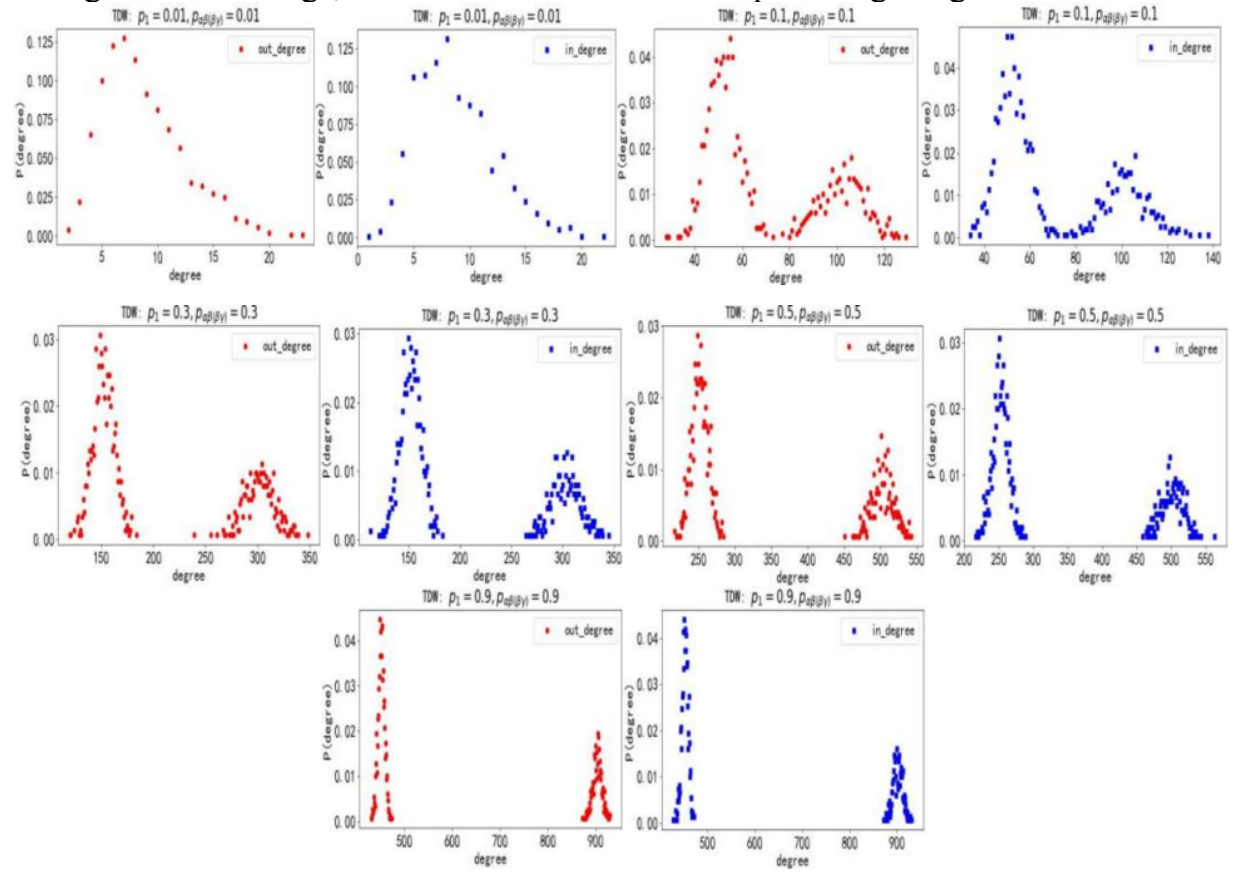

Figure 4. Degree distribution of three-layer directed network TDW.

\subsubsection{Different Probability of Directed Connection Between Layers.}

(1) For the three-layer directed network TDB, when $p_{\alpha \beta}+p_{\beta \gamma} \neq 1$, and $m=2, p_{\alpha \beta}=$ $10^{-3}, p_{\beta \gamma}=0.1,0.3,0.5,0.9$, the experimental results are shown in table 6 and figure 5 . 
Table 6. Degree distribution characteristics of three-layer directed network TDB.

\begin{tabular}{c|cccccccccc}
\hline Parameter & $p_{\beta \gamma}$ & $\left\langle k_{\alpha}^{\text {in }}\right\rangle$ & $\left\langle k_{\alpha}^{\text {out }}\right\rangle$ & $\left\langle k_{\beta}^{\text {in }}\right\rangle$ & $\left\langle k_{\beta}^{\text {out }}\right\rangle$ & $\left\langle k_{\gamma}^{\text {in }}\right\rangle$ & $\left\langle k_{\gamma}^{\text {out }}\right\rangle$ & $\left\langle k^{\text {in }}\right\rangle$ & $\left\langle k^{\text {out }}\right\rangle$ & $\begin{array}{c}\text { Degree } \\
\text { Distribution }\end{array}$ \\
\hline \multirow{2}{*}{$\begin{array}{c}\text { Network } \\
\text { TDB }\end{array}$} & 0.1 & 2.5 & 2.5 & 52.7 & 52.3 & 51.8 & 52.2 & 35.7 & 35.7 & power law \\
& 0.3 & 2.5 & 2.5 & 152.5 & 152.5 & 152 & 152 & 102.3 & 102.3 & and \\
& 0.5 & 2.5 & 2.5 & 252.9 & 252.2 & 251.7 & 252.3 & 169 & 169 & unimodal \\
& 0.9 & 2.5 & 2.5 & 452.2 & 452.7 & 452.2 & 451.8 & 302.5 & 302.5 & \\
\hline
\end{tabular}
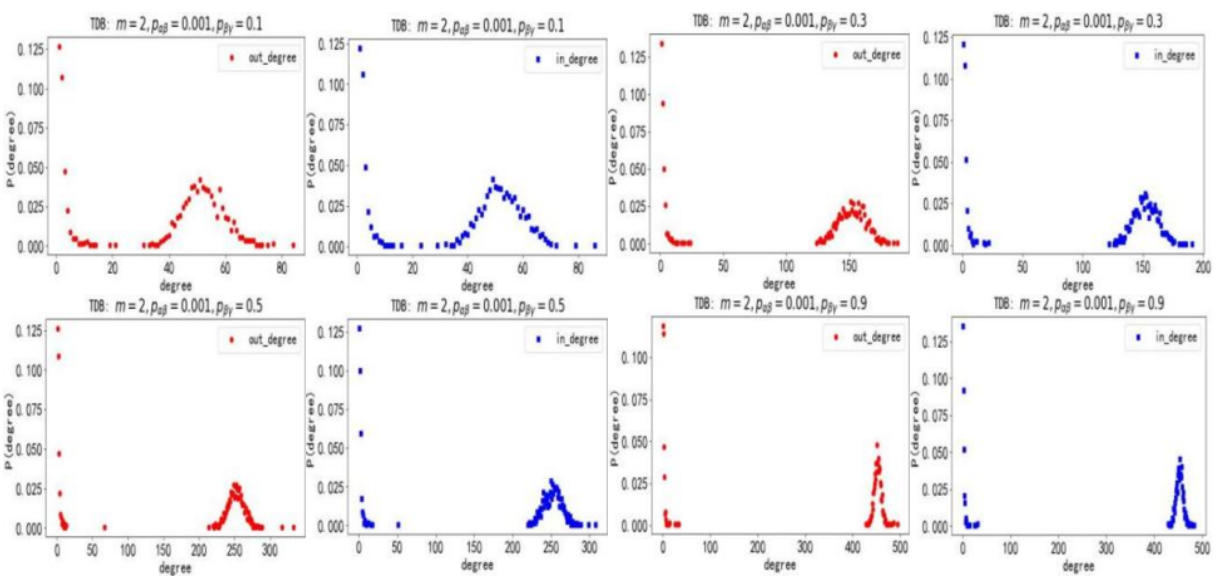

Figure 5. Degree distribution of three-layer directed network TDB.

It can be seen from table 6 and figure 5 that the out-degree distribution and in-degree distribution of the three-layer directed network TDB simultaneously show power law and unimodal characteristics. Because $p_{\alpha \beta}=0.001$, the value is very small, the average out-degree and the average in-degree value of the single-layer directed network $\overrightarrow{G_{\alpha}}$ is relatively small. At this time, the out-degree distribution and in-degree distribution of the network show power-law characteristics. However, the average out-degree and average in-degree values of the directed networks $\overrightarrow{G_{\beta}}$ and $\overrightarrow{G_{\gamma}}$ of the individual layers increase with the increase of the inter-layer connection probability, and they are almost equal, so they fall on the single peak on the right after the overlap.

(2) Discuss the case where the intra-layer connection probability and the inter-layer connection probability are not the same in the three three-layer directed networks. The intra-layer connection probability of the three-layer directed network TDE and the threelayer directed network TDW is $p_{1}=0.5$. The value range of connection probability $p_{\alpha \beta}\left(p_{\beta \gamma}\right)$ is $(0,1)$, and the sum of the intra-layer connection probability and the interlayer connection probability is 1 . In the directed network TDB, $m=2$, the value of the inter-layer connection probability is the same as the other two three-layer directed network is the same. The experimental results are shown in table 7 and figure 6 . 
Table 7. Degree distribution of three kinds of three-layer directed networks.

\begin{tabular}{|c|c|c|c|c|c|c|c|c|c|c|c|}
\hline Parameter & $p_{\alpha \beta}$ & $p_{\beta \gamma}$ & $\left\langle k_{\alpha}^{i n}\right\rangle$ & $\left\langle k_{\alpha}^{\text {out }}\right\rangle$ & $\left\langle k_{\beta}^{i n}\right\rangle$ & $\left\langle k_{\beta}^{\text {out }}\right\rangle$ & $\left\langle k_{\gamma}^{i n}\right\rangle$ & $\left\langle k_{\gamma}^{\text {out }}\right\rangle$ & $\left\langle k^{i n}\right\rangle$ & $\left\langle k^{\text {out }}\right\rangle$ & $\begin{array}{c}\text { Degree } \\
\text { distribution }\end{array}$ \\
\hline & 0.1 & 0.9 & 299.4 & 299.6 & 749.4 & 749.6 & 699.6 & 699.4 & 582.8 & 582.8 & trimodal \\
\hline Network & 0.3 & 0.7 & 399.7 & 399.3 & 749.2 & 749.8 & 599.7 & 599.4 & 582.8 & 582.8 & trimodal \\
\hline \multirow[t]{3}{*}{ TDE } & 0.9 & 0.1 & 699.3 & 699.7 & 749.8 & 749.2 & 299.4 & 299.6 & 582.8 & 582.8 & trimodal \\
\hline & 0.5 & 0.5 & 499 & 500 & 749.4 & 749.6 & 500.1 & 498.9 & 582.8 & 582.8 & bimodal \\
\hline & 0.1 & 0.9 & 52.1 & 51.9 & 502 & 502 & 451.9 & 452.1 & 335.3 & 335.3 & trimodal \\
\hline Network & 0.3 & 0.7 & 151.8 & 152 & 502 & 502 & 352 & 351.7 & 335.3 & 335.3 & trimodal \\
\hline \multirow[t]{3}{*}{ TDB } & 0.9 & 0.1 & 451.8 & 452.2 & 501.9 & 502 & 51.7 & 52.3 & 335.3 & 335.3 & trimodal \\
\hline & 0.5 & 0.5 & 251.9 & 252.2 & 502 & 502 & 252 & 251.9 & 335.3 & 335.3 & bimodal \\
\hline & 0.1 & 0.9 & 52.2 & 51.8 & 502.1 & 501.9 & 451.7 & 452.3 & 335.3 & 335.3 & trimodal \\
\hline Network & 0.3 & 0.7 & 152.4 & 151.6 & 501.6 & 502.4 & 352 & 352 & 335.3 & 335.3 & trimodal \\
\hline \multirow[t]{2}{*}{ TDW } & 0.9 & 0.1 & 451.9 & 452.1 & 501.9 & 502.1 & 51.7 & 52.2 & 335.3 & 335.3 & trimodal \\
\hline & 0.5 & 0.5 & 252 & 252 & 503 & 501 & 251 & 252 & 335.3 & 335.3 & bimodal \\
\hline
\end{tabular}

From table 7 and figure 6, when the inter-layer connection probabilities are different and the value range is $(0,1)$ and $p_{\alpha \beta}+p_{\beta \gamma}=1$, the average out-degree and average indegree values of the directed networks $\overrightarrow{G_{\alpha}}, \overrightarrow{G_{\beta}}$ and $\overrightarrow{G_{\gamma}}$ of the individual layers are different. When the network's out-degree distribution and in-degree distribution show trimodal characteristics, and when the inter-layer connection probability is $p_{\alpha \beta}=0.3$ and $p_{\beta \gamma}=0.7$ the trimodal are more obvious. In particular, when $p_{\alpha \beta}=p_{\beta \gamma}=0.5$, the outdegree distributions and in-degree distributions show bimodal characteristics.
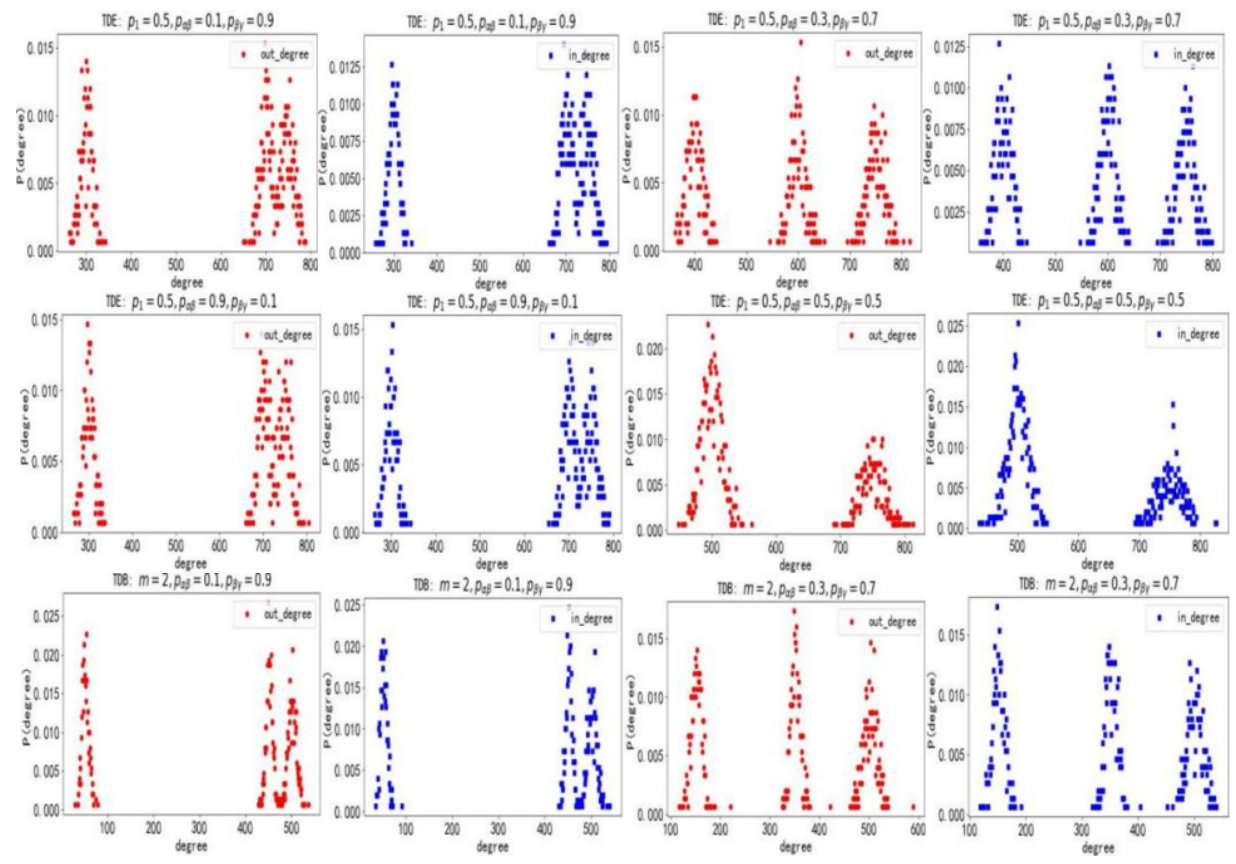

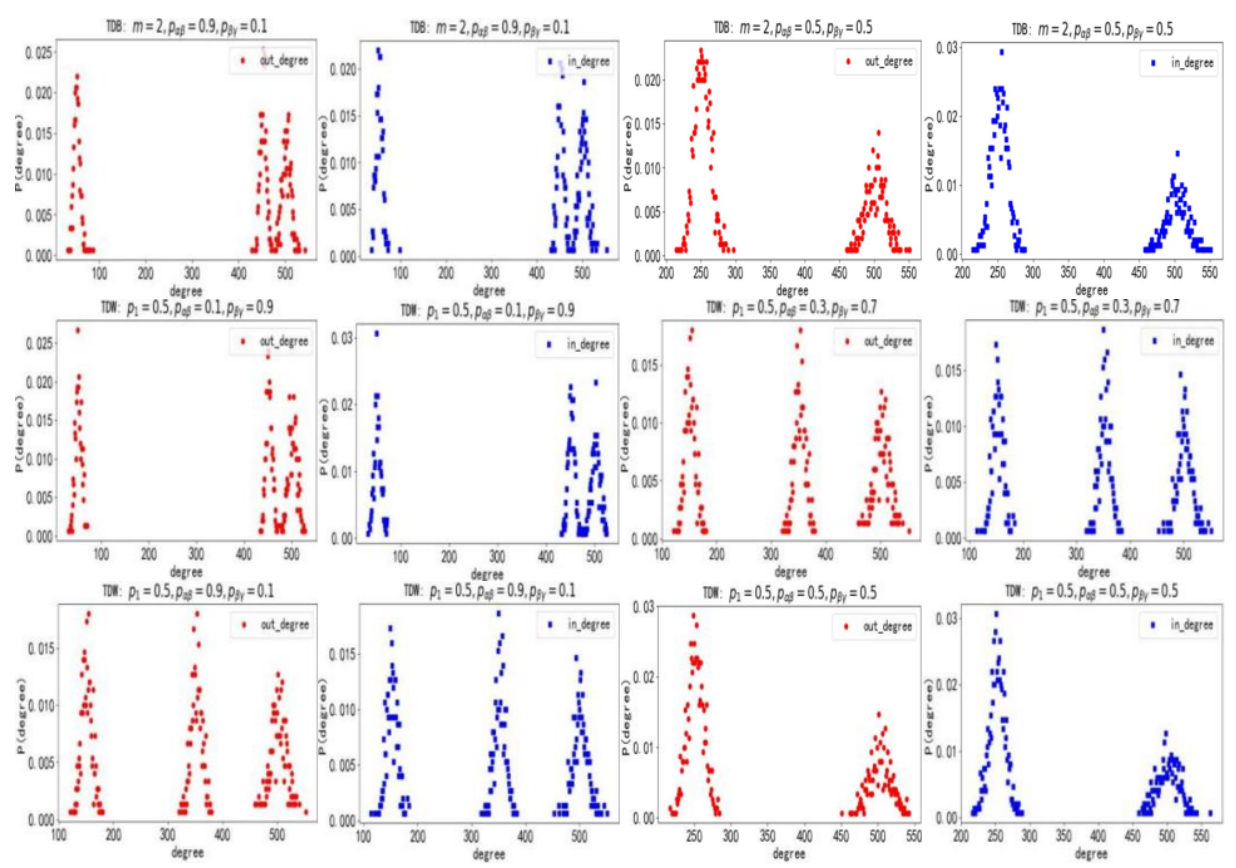

Figure 6. Degree distribution of three kinds of three-layer directed networks.

Through all the above experiments, it can be obtained that the intra-layer connection probability of a single-layer directed network has little effect on the entire network, but when the intra-layer connection probability is large and the inter-layer connection probability is small, the out-degree distribution and in-degree distribution of the threelayer directed network show unimodal characteristics; when the inter-layer connection probability is the same and the value is large, the out-degree distribution and in-degree distribution of the three-layer directed network show bimodal characteristics. At this time, the average out-degree and average in-degree values of directed $\overrightarrow{G_{\alpha}}$ and $\overrightarrow{G_{\gamma}}$ of the individual layer are basically the same, which becomes one of the peaks in the degree distribution graph, and the degree value of the individual layer $\overrightarrow{G_{\beta}}$ becomes the other peak in the degree distribution graph.; when the inter-layer connection probabilities are not the same, the individual layer is directed The average out-degree and average indegree values of networks $\overrightarrow{G_{\alpha}}, \overrightarrow{G_{\beta}}$, and $\overrightarrow{G_{\gamma}}$ are different. At this time, the network exhibits a three-peak characteristic; in a three-layer directed network TDW, when the inter-layer connection probability is different and the value of $p_{\alpha \beta}$ is very small, the value of $p_{\beta \gamma}$ gradually increases, and the out-degree distribution and in-degree distribution of the network appear power law and unimodal co-occurrence. 


\section{Conclusion}

Based on three types of classic directed network construction algorithms, this paper uses the inter-layer random connection mechanism to construct three three-layer directed networks, and analyzes the degree distribution characteristics on the basis of the network model. The conclusions are as follows: 1) When the inter-layer connection When the probability is equal and the value is small, the out-degree distribution and in-degree distribution show unimodal characteristics, and when the value is larger, the out-degree distribution and in-degree distribution show bimodal characteristics. 2) When the interlayer connection probabilities are not equal, the out-degree distribution and in-degree distribution of the network present the characteristics of three peaks, and when the probability of inter-layer connection is small, the network TDB will have both power law and unimodal coexistence. In the subsequent research, we will continue to improve the algorithm, increase the complexity of the inter-layer connection algorithm, explore the changes in the network structure, and derive the theoretical formula of the in-degree distribution.

\section{Acknowledgments}

This work is supported by the Science Found of Qinghai Province (Grant No. 2020-GX112), and the Key Laboratory of Tibetan Information Processing, Ministry of Education, and the Tibetan Information Processing and Machine Translation Key Laboratory of Qinghai Province (Grant No. 2020-ZJ-Y05).

\section{References}

[1] Wang X F, Li X and Chen G R 2012 Network Science: An Introduction (Beijing: Higher Education Press)

[2] Lewis T G 2009 Network Science - Theory and Applications (Wiley Publishing)

[3] Barabasi A L and Albert R 1999 Science 286(5439) 509-512

[4] P Erdős and A Rényi 1959 Publicationes Mathematicae 6 290-297

[5] Guo J L 2013 Complex Network and Dynamic Evolution Model of Human Behavior (Beijing: Science Press)

[6] Watts D J and Strogatz S H 1998 Nature 393(6684) 440-442

[7] Fang J Q 2016 Complex Systems and Complexity Science 13(1) 40-47

[8] He D R 2009 Complex Systems and Complex Networks (Beijing: Higher Education Press)

[9] Xian X P and Wu T 2016 Industrial \& Science Tribune 15(019) 80-81

[10] Lu J A 2015 Modern Physics 027(004) 3-8

[11] Wu Z N, Di Z R and Fan Y 2021 Journal of University of Electronic Science and Technology of China 50(01) 106-120

[12] Chen X M, Li Y Z and Shen Q 2019 Journal of Computer Applications 39(10) 3079-3087

[13] Shen A Z, Guo J L and Suo Q 2017 Application Research of Computer 12 3628-3631

[14] Min B, Yi S D, Lee K M 2013 Phys.rev.e 89(4) 1600-1618

[15] Ma H Y, Xiao Y Z, Zhao H X 2020 Complex Systems and Complexity Science 17(04) 16-29

[16] D H Shi M 2011 Theory of Network Degree Distributions (Beijing: Higher Education Press)

[17] Liu D W, Yang W F, Wang H Y 2018 Journal of Chinese Computer Systems 39(10) 71-75

[18] Chen X, Huang S G and Qin Li 2015 Journal of Computer Applications 35(3) 638-642

[19] Liu D H, Zhang B F, Zou G B 2016 Computer Applications and Software 7 129-133

[20] Xu Z P, Fu X C 2019 Acta Mathematica Scientia v.39(06) 255-274 